\title{
What are the potential benefits and harms of exercise programs for patients with ankylosing spondylitis? A Cochrane review summary with commentary
}

\author{
İlke Coşkun Benlidayı (10) \\ Department of Physical Medicine and Rehabilitation, Çukurova University Faculty of Medicine, Adana, Turkey \\ Received: February 08, 2020 Accepted: February 17, 2020 Published online: March 03, 2020
}

The aim of this commentary is to discuss in a rehabilitation perspective the published Cochrane Review "Exercise programmes for ankylosing spondylitis" ${ }^{[1]}$ by Regnaux, Davergne, Palazzo, Roren, Rannou, Boutron, Lefevre-Colau under the direct supervision of Cochrane Musculoskeletal Group. This Cochrane Corner is produced in agreement with the Turkish Journal of Physical Medicine and Rehabilitation by Cochrane Rehabilitation.

Ankylosing spondylitis (AS) is an inflammatory rheumatic disease with an incidence rate of $0.5-4 / 100,000$ individuals per year. ${ }^{[2]}$ Major symptoms include inflammatory back pain, stiffness, and fatigue. It is characterized by the inflammation of the axial skeleton. On the other hand, peripheral joint involvement and/or extra-articular manifestations may be also seen throughout the disease course.

Over the past decades, there have been dramatic advances in pharmacological treatment strategies for AS. Non-steroidal antirheumatic drugs (NSAIDs) and biological disease-modifying antirheumatic drugs (bDMARDs) have been the mainstay of pharmacological therapy. However, nonpharmacological treatment options including exercise programs, have still remained essential for relieving pain and stiffness along with improving function and health-related quality of life. Exercise refers to planned and structured physical activity, which is repeated over a time period. ${ }^{[3]}$ Exercise programs for AS vary in terms of type, duration, frequency, and intensity. In this regard, it is of paramount value to systematically review the current knowledge/evidence derived from randomized-controlled trials (RCTs) on exercise programs in AS. A Cochrane Review synthetized evidence regarding the beneficial and harmful effects of exercise programs in individuals with AS. ${ }^{[1]}$

\section{Exercise programmes for ankylosing spondylitis} (Regnaux et al., 2019) $)^{[1]}$

\section{What is the aim of the Cochrane review?}

The aim of the Cochrane review is to identify the potential benefits and harms of exercise programs in patients with AS by reviewing the current evidence derived from RCTs.

\section{What was studied in the Cochrane review?}

The population studied in the Cochrane review included adult patients ( $\geq 18$ years) diagnosed with AS with the exclusion of participants with non-radiographic axial spondyloarthritis. The interventions studied were all interventions including any type of exercises (land- or water-based) such as aerobic, strengthening, mobilizing, flexibility, stretching, balance exercises, or functional training, and specific programs (Tai chi or Yoga) carried out in any setting/location with any type of delivery including individually, in groups or mixed. General activities such as walking or swimming which

Corresponding author: İlke Coşkun Benlidayı, MD. Çukurova Üniversitesi Tıp Fakültesi, Fiziksel Tıp ve Rehabilitasyon Anabilim Dalı, 01330 Sarıçam, Adana, Türkiye. e-mail: icbenlidayi@hotmail.com 
were not conforming to the definition of exercise ${ }^{[3]}$ were excluded. The comparators were usual care or no intervention/exercise. The experimental groups received exercise programs either alone (monomodal) or in combination with other interventions. The primary outcomes were physical function, pain, patient global assessment of disease activity, spinal mobility, fatigue, and safety. The secondary outcomes were quality of life, acute-phase reactants, physician global assessment, peripheral joints/entheses-pain, swelling, and tenderness. An outcome improvement of $>15 \%$ for an absolute change and of $>20 \%$ for a relative change was considered an important clinical benefit.

\section{Search methodology and up-to-dateness of the Cochrane review}

The authors searched through electronic databases and other sources by using free text words and controlled vocabulary based on the keywords (or their synonyms) of "exercise" AND "spondyloarthritis". The following electronic databases were searched up to 14 December 2018: CENTRAL, MEDLINE Ovid, Embase Ovid, CINAHL EBSCO, PEDro, and Scopus. The authors also searched for related systematic reviews through the Cochrane Database of Systematic Reviews and the Database of Abstracts of Reviews of Effect. Other sources were the reference lists of included articles, Google, Google Scholar, proceedings of the American College of Rheumatology, European League Against Rheumatism, Osteoarthritis Research Society International and the clinical trial registry platforms (ClinicalTrials.gov and World Health Organization International Clinical Trials Registry Platform).

\section{What are the main results of the review?}

The review included 14 RCTs $(n=1,579)$ with a parallel-group design. The median age of the patients was 45 (range, 39 to 47 ) years and the majority of the patients (median 70\%) were males. The median exercise program duration and session duration were 12 weeks and $60 \mathrm{~min}$, respectively. Of 14 included trials, nine tailored exercise programs alone to the experimental group, while the rest investigated exercise programs in combination with other interventions. In the majority of the studies, exercise programs were land-based, supervised, and included strengthening component.

Immediately after completion of the intervention, comparison of exercise programs with no intervention/ exercise revealed that;

- Exercise programs provided an absolute reduction of $13 \%$ (95\% confidence interval [CI] 17 to $9 \%$ ) in physical function score as measured using the Bath Ankylosing Spondylitis Functional Index (BASFI) in patients with AS, indicating the probability of a slight improvement in this outcome. Although the absolute improvement was below the threshold for an important clinical benefit, the estimate included both clinically important and clinically unimportant effects. The quality of evidence was moderate ( 7 trials, $n=312$ ).

- Pain scores measured by visual analog scale (VAS) decreased significantly by an absolute reduction of $21 \%$ (95\% CI: 36 to 6\%), indicating that exercise might cause a reduction in pain. There was an important clinical meaningful benefit. However, the quality of evidence was low (6 trials, $\mathrm{n}=288$ ).

- Exercise programs provided a significant absolute reduction of $9 \%$ (95\% CI: 13 to 5\%) in patient global assessment of disease activity as measured using the Bath Ankylosing Spondylitis Disease Activity Index (BASDAI) with no important clinical meaningful benefit. The quality of evidence was moderate ( 6 trials, $\mathrm{n}=262$ ).

- The change in spinal mobility assessed by the Schober's test did not significantly differ between the study groups ( 3 trials, $n=136$ ). On the other hand, spinal mobility evaluated by the Bath Ankylosing Spondylitis Metrology Index (BASMI) improved in patients who exercised by an absolute reduction of $7 \%$ (95\% CI: 13 to $1 \%$ ). However, there was no important clinical meaningful benefit and the quality of evidence was very low ( 5 trials, $n=232$ ).

- Fatigue (as measured using VAS) decreased significantly with exercise by $14 \%$ (95\% CI: 27 to $1 \%$ ). The absolute change was below the threshold for an important clinical improvement and the quality of evidence remained very low ( 2 trials, $n=72$ ).

Immediately after completion of the intervention, comparison of exercise programs with usual care revealed the followings:

- Physical function improved by a reduction of $4 \%$ (95\% CI: 6 to 2\%) in BASFI scale in patients who exercised albeit with no important clinical meaningful benefit. The quality of evidence was moderate ( 5 trials, $\mathrm{n}=1,068$ ).

- Exercise provided reduction in pain scores by 5\% (95\% CI: 9 to 1\%). However, the benefit was not clinically meaningful. The quality of evidence was moderate ( 2 trials, $\mathrm{n}=911$ ).

- Although patient global assessment of disease activity reduced significantly with exercise 
[MD (mean difference) -0.7, 95\% CI: -1.3 to -0.1$]$ with a reduction of $7 \%$ ( $95 \%$ CI: 13 to $1 \%$ ) in BASDAI scores, no important clinical meaningful improvement was observed considering the clinically important threshold as $15 \%$ absolute change. The quality of evidence remained low ( 5 trials, $\mathrm{n}=1,068$ ).

- The results were inconclusive in terms of spinal mobility. No important clinical meaningful benefit was achieved, and the quality of evidence was very low ( 2 trials, $\mathrm{n}=85$ ).

- Fatigue was not measured in any of the studies.

\section{Safety:}

There is uncertainty on whether exercises are harmful or not as the quality of evidence was assessed as very low; only two trials $(\mathrm{n}=110)$ reported data on adverse effects, the number of which was very low (one exercise group participant with increased back pain).

\section{How did the authors conclude on the evidence?}

- There is moderate-to-low-quality evidence suggesting that exercise programs, compared to no intervention, may provide clinically important pain reduction, probably improve physical function and reduce patient global assessment of disease activity slightly when measured at the end of the intervention. There is uncertainty regarding the impact of exercise programs on spinal mobility and fatigue.

- Compared to usual care at the end of interventions, moderate-to-low-quality evidence supports that exercise programs probably make little or no difference in physical function and pain, may also have little or no impact on patient global assessment of disease activity. There is uncertainty regarding the effect of exercise programs on spinal mobility.

- There is no clear evidence to conclude that exercise programs can lead to more adverse effects.

\section{What are the implications of the Cochrane evidence for practice in rehabilitation?}

Management of AS is a complex issue. Management protocols should not only focus on pharmacological treatment, but should also take non-pharmacological therapeutic options into account. Exercise is regarded as an important component of non-pharmacological treatment for AS. Supervised and/or unsupervised exercises are recommended for the treatment of patients with AS. ${ }^{[4,5]}$ The main goals of exercise are to increase spinal range of motion, improve posture, enhance physical function, relieve pain, decrease disease activity, and promote general health and well-being. Different types of exercises such as stretching, strengthening and cardiopulmonary/ functional fitness can be applied..$^{[5]}$ The type, duration, and intensity of the exercise regimens vary depending on the individual's needs. It is also essential to pay meticulous attention on the potential harms of exercise programs in this particular patient group.

In the light of these data, the topic and the related aim of the present Cochrane review by Regnaux et al. ${ }^{[1]}$ are of great value. The results of the Cochrane review support the benefits of exercise programs, versus no intervention, on physical function and patient global assessment of disease activity (albeit slightly with no clinically important beneficial effect for both) as well as on pain with a significant clinically meaningful reduction based on moderate-to-low-quality evidence. On the other hand, the authors concluded that exercise programs, compared to usual care (self-management, medication, physiotherapy), have little/no effect on these parameters.

The current Cochrane review ${ }^{[1]}$ provided rehabilitation professionals with some evidence regarding the role of exercise in patients with AS. Implementing exercise programs in the management practices would probably or might be beneficial in terms of pain relief and might slightly improve function and patient-perceived disease activity compared to no intervention. Exercise, therefore, potentially stands as an essential non-pharmacological treatment option for patients with AS. Low-quality evidence on some outcomes could be a driving force for rehabilitation professionals to continue with research on the topic, since it is likely that evidence may change with future research.

The Cochrane review ${ }^{[1]}$ provides a clear insight about the duration and frequency of exercise programs in AS. However, the included trials evaluated different types of exercises and the exercise components (materials used, provider, etc.) are incompletely described. Therefore, the review was unable to draw conclusion on these points. In addition, the authors were unable to obtain clear evidence regarding the role of exercise programs in improving spinal mobility and fatigue. The evidence on potential harms of exercise regimens remained scarce, as well. Since none of the included RCTs evaluated the effectiveness of exercise programs in patients with newly diagnosed/early AS, the results of the present review should be applied with caution to patients with shorter disease duration. Thus, 
further researches among patients with shorter disease duration may change the current evidence. Finally, most of the patients in this review were on NSAID therapy and therefore the review could not provide any comparative data regarding the effectiveness of exercise programs in patients on biologic therapy versus those on NSAIDs/conventional synthetic DMARDs. Future trials taking these remaining issues into consideration would be of value to improve the current knowledge on exercise programs in AS.

\section{Acknowledgement}

The author thanks Cochrane Rehabilitation and Cochrane Musculoskeletal Group for reviewing the contents of the Cochrane Corner.

\section{Declaration of conflicting interests}

The author declared no conflicts of interest with respect to the authorship and/or publication of this article.

\section{REFERENCES}

1. Regnaux JP, Davergne T, Palazzo C, Roren A, Rannou F, Boutron I, et al. Exercise programmes for ankylosing spondylitis. Cochrane Database Syst Rev 2019;10:CD011321. doi: 10.1002/14651858.CD011321.

2. Braun J, Sieper J. Ankylosing spondylitis. Lancet 2007;369:1379-90.

3. Bouchard C, Blair S, Haskell W. Physical Activity and Health. Champaign, IL: Human Kinetics Inc; 2012.

4. Ward MM, Deodhar A, Gensler LS, Dubreuil M, Yu D, Khan MA, et al. 2019 Update of the American College of Rheumatology/Spondylitis Association of America/ Spondyloarthritis Research and Treatment Network Recommendations for the Treatment of Ankylosing Spondylitis and Nonradiographic Axial Spondyloarthritis. Arthritis Rheumatol 2019;71:1599-613.

5. Millner JR, Barron JS, Beinke KM, Butterworth RH, Chasle BE, Dutton LJ, et al. Exercise for ankylosing spondylitis: An evidence-based consensus statement. Semin Arthritis Rheum 2016;45:411-27. 